\title{
FOXP2 as a molecular window into speech and language
}

\author{
Simon E. Fisher ${ }^{1}$ and Constance Scharff ${ }^{2}$ \\ ${ }^{1}$ Wellcome Trust Centre for Human Genetics, University of Oxford, Roosevelt Drive, Oxford, OX3 7BN, UK \\ ${ }^{2}$ Department of Animal Behavior, Institute of Biology, Freie Universität, Berlin, Germany
}

\begin{abstract}
Rare mutations of the FOXP2 transcription factor gene cause a monogenic syndrome characterized by impaired speech development and linguistic deficits. Recent genomic investigations indicate that its downstream neural targets make broader impacts on common language impairments, bridging clinically distinct disorders. Moreover, the striking conservation of both FoxP2 sequence and neural expression in different vertebrates facilitates the use of animal models to study ancestral pathways that have been recruited towards human speech and language. Intriguingly, reduced FoxP2 dosage yields abnormal synaptic plasticity and impaired motor-skill learning in mice, and disrupts vocal learning in songbirds. Converging data indicate that Foxp2 is important for modulating the plasticity of relevant neural circuits. This body of research represents the first functional genetic forays into neural mechanisms contributing to human spoken language.
\end{abstract}

\section{A genetic perspective on speech and language}

The anatomical, neural and cognitive bases of speech and language have been open to investigation for some years. Only recently have modern genetic tools been brought to bear on this fascinating aspect of being human [1]. A novel molecular perspective is emerging, driven by isolation of genomic variants that are correlated with disturbances in speech, language and/or reading [2,3]. One finding at the heart of this new wave of research was the discovery of FOXP2 mutations that cause a monogenic speech and language disorder $[4,5]$. Given adequate environmental input, children normally acquire highly proficient spoken language without conscious effort or formal tuition [6]. By contrast, individuals carrying heterozygous FOXP2 mutations have difficulties mastering complex sequences of mouth movements underlying speech (developmental verbal dyspraxia), and have impaired expressive and receptive language, whereas other aspects of cognition and development are relatively spared [7] (Box 1).

A role for FOXP2 first became apparent based on investigations of a multigenerational pedigree (the KE family) in which all affected members inherited a missense mutation (R553H) disturbing the DNA-binding domain of the encoded protein [4]. Subsequent studies identified additional cases of FOXP2 disruption (Box 1), but indicated that these are rare. For example, it has been estimated that only $\sim 1$ in 50 people diagnosed with developmental verbal dyspraxia carry aetiological point mutations [5].

Corresponding author: Fisher, S.E. (simon.fisher@well.ox.ac.uk).
Nevertheless, the gene provides a molecular entry point into neurogenetic mechanisms facilitating human spoken language [8], opening up innovative avenues of investigation that are already proving fruitful.

Two characteristics make FOXP2 especially amenable for this purpose. First, the gene encodes a regulatory protein, belonging to the Forkhead-box group of transcription factors [9]; hence, functional genomics can identify other elements of the pathways in which it participates, including targets that it regulates. Second, FoxP2 orthologues exist in highly similar forms in many vertebrates, showing comparable neural expression patterns; in humans, monkeys, mice, rats, birds, crocodiles and zebrafish, FoxP2 is consistently expressed in distributed circuits involving (among others) the cortex, basal ganglia (BG), thalamus and cerebellum [10-17]. Such remarkable conservation has led some to posit that the link between FOXP2 and language is weak [18]. This claim is based on the misconception that a unique trait requires genes that are exclusive to the species and organs that exhibit it. By contrast, we argue that because the human faculty for spoken language is built on multiple traits that predate its emergence, its evolution is unlikely to be explained in terms of a single human-specific or brain-specific molecular agent [1,19]. Here, we update findings [20] regarding FoxP2 function in a range of species (including human, mice and songbirds) and reveal how they help elucidate the contributions of this gene to speech and language.

\section{Building bridges between distinct language disorders}

Although mutations of FOXP2 itself are rare, it is plausible that the downstream pathways it regulates could have broader relevance for common language impairments (Box 2). Recently, Vernes et al. [21] employed unbiased genomic screening to isolate direct neural targets of FOXP2 that might be implicated in language disorders. The strategy exploited chromatin-immunoprecipitation (ChIP) with FOXP2-recognizing antibodies to purify genomic fragments that the protein binds in native chromatin of human neuronal cell models, followed by shotgunsequencing to establish identity of enriched fragments. This technique uncovered a FOXP2-bound fragment in the first intron of CNTNAP2, the gene encoding 'contactinassociated protein-like 2', a member of the neurexin superfamily (Figure 1). Neurexins are neuronal transmembrane proteins involved in cell adhesion; classical neurexins function primarily as polymorphic synaptic receptors, whereas contactin-associated proteins have 


\section{Box 1. FOXP2 mutations in humans}

The discovery of human FOXP2 and its potential role in speech and language resulted from positional cloning studies of a large multigenerational pedigree (the KE family) [4]. Approximately half (15 people) of the family members had difficulties in sequencing mouth movements, impairing speech (developmental verbal dyspraxia), along with wide-ranging oral and written language deficits, affecting both expressive and receptive skills [7]. Effects on nonverbal cognition were less severe and unlikely to be central [7]. After mapping the locus responsible to chromosome 7q31 [74], it was found that all affected individuals carried a heterozygous point mutation in FOXP2, yielding an arginine-to-histidine substitution at a crucial residue $(\mathrm{R} 553 \mathrm{H})$ in the DNA-binding domain of the encoded protein [4]. An unrelated child with similar deficits had a de novo balanced translocation [75] directly disrupting the FOXP2 locus [4].

Subsequently, mutation screening in a panel of 49 probands with a diagnosis of developmental verbal dyspraxia identified a novel aetiological point mutation [5]. In this case, the change was a heterozygous nonsense mutation (R328X) predicted to severely truncate the FOXP2 protein. Although there was no specific requirement for familial clustering when selecting the panel for this study, the proband in question had an affected sister and mother, both of whom also carried the R328X mutation [5]. Several heterozygous or hemizygous chromosomal rearrangements involving FOXP2 have since been reported, including translocations and deletions [43-46]. People who have Silver-Russell syndrome associated with maternal uniparental disomy of chromosome 7 sometimes show speech difficulties, which might correlate with reduced FOXP2 expression [43]. One study proposed that the gene is maternally imprinted, primarily based on absence of a paternal copy in people with verbal dyspraxia [43]. However, this hypothesis is inconsistent with independent observations of maternally inherited mutations, deletions and translocations in other reports $[4,5,45,46]$. It seems likely that disruption of one FOXP2 copy (whether maternal or paternal) is sufficient to derail speech and language development.

There is continued debate about the relationship between the verbal dyspraxia and the co-occurring linguistic impairments observed in people with FOXP2 disruptions. Although some accounts propose that the broader language deficits in these cases are purely secondary to core problems with orofacial motor control, others note that the gene could have pleiotropic effects, impacting on neural pathways underlying multiple aspects of speech and language (for a review, see Ref. [3]).

been implicated in neuron-glia interactions [22]. CNTNAP2 is implicated in neuronal recognition, cell adhesion and localization and maintenance of voltagegated potassium channels [22]. Further experiments in transfected human neuron-like cells indicated that FOXP2 downregulates CNTNAP2 expression [21]. Both genes are expressed in the developing human cerebral cortex $[10,13,23]$, where they show complementary patterns; cortical layers with the highest FOXP2 expression have the lowest CNTNAP2 levels [21].

CNTNAP2 represented a promising candidate for involvement in language impairments. A genome-wide investigation of gene expression in midgestation human fetal brains observed strong CNTNAP2 enrichment in frontal gray matter of the developing cerebral cortex [23]. Moreover, a homozygous CNTNAP2 mutation, predicted to yield a truncated nonfunctional protein, has been found in a family with cortical dysplasia (malformations of the cortex due to abnormal development in utero) and focal epilepsy, accompanied by language regression and autistic characteristics [24].

Therefore, Vernes et al. [21] genotyped single nucleotide polymorphisms (SNPs) spanning CNTNAP2 in 184 small

\section{Box 2. Defining language disorders}

The process of speech and language acquisition is extraordinarily complex, yet we often take it for granted. With adequate opportunity, the overwhelming majority of children develop adept spoken language skills rapidly during the first few years of life. Sometimes when a child fails to acquire normal language this occurs as a secondary consequence of a physical or neurological problem such as mental retardation, deafness or cleft-lip or cleftpalate. However, significant speech and/or language difficulties in a subset of children remain unexplained [6]. Robust diagnosis of this type of developmental disorder can be challenging, being partly based on exclusionary criteria (i.e. absence of particular symptoms) [76].

Some forms of speech and language disorder, such as the one caused by mutation of FOXP2, involve problems with controlling fine co-ordinated movement sequences of the mouth, tongue, lips and soft palate, yielding a diagnosis of development verbal dyspraxia (also referred to as childhood apraxia of speech). However, it is more common to find children with language impairments that occur in absence of these kinds of overt articulation problems.

Many such children are given a diagnosis of SLI, typically defined as a significant discrepancy between their verbal and non-verbal abilities, assessed by standardized batteries of tests. An epidemiological study of preschool children (aged 5-6 years) in the US has estimated a prevalence of up to 7\% [77]. In practice, a diagnosis of SLI encompasses a large degree of heterogeneity; there can be considerable variation in profile (i.e. which aspects of language are impaired) and severity of deficits observed among different affected people, or even in the same individual at different ages [3]. SLI can be divided into categories such as phonological disorder, expressive disorder and mixed (expressive and receptive) disorder, but the biological validity of this classification scheme remains open to question [78]. Regardless of these diagnostic issues, SLI has been consistently shown to be highly heritable [6]. The underlying genetic architecture of typical SLI seems complex and multifactorial [76], but genome-wide linkage scans have highlighted several chromosomal regions that are likely to harbour risk factors $[25,26,79]$. Some of these studies have overcome the complexities of defining the disorder by identifying heritable behavioural markers that can act as robust endophenotypes [76]. Notably, FOXP2 mutations or associations have not been detected in common forms of SLI [80].

families with typical forms of specific language impairment (SLI) defined as unexplained deficits in expressive and/or receptive language despite adequate intelligence and environmental input (Box 2). This set of families was previously ascertained from multiple sites in the UK by the SLI Consortium (http://www.well.ox.ac.uk/monaco/ SLI/SLIC.shtml) [25,26]. Quantitative family-based association analyses of CNTNAP2 markers indicated that intronic SNPs between exons 13-15 were significantly associated with deficits in the ability to repeat nonsensewords, a robust endophenotype of SLI [21]. This same region showed association with clinical language measures, although this was weaker than the endophenotype. Multi-marker analyses of the associated SNPs identified a common risk haplotype negatively influencing nonsense-word repetition [21] (Figure 1).

As well as establishing connections between rare monogenic disorders and common complex traits, these data indicate links between diagnostic categories that are traditionally considered as clinically distinct. Earlier investigations implicated genomic CNTNAP2 variants in autism, a disorder in which communication deficits are accompanied by impaired social interactions and rigid and 
(a)



(b)

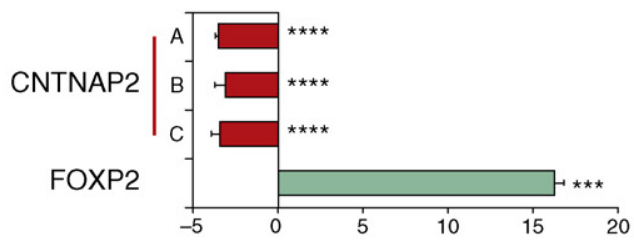

Mean $\log _{2}$ ratio of normalized mRNA (c)

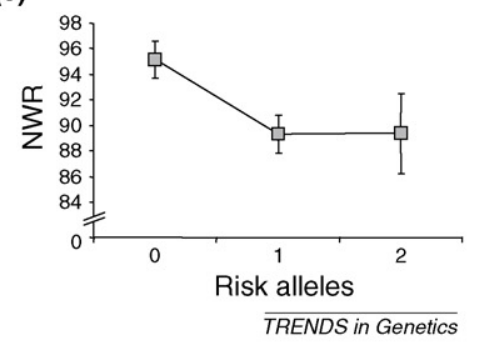

Figure 1. Functional genetic bridges between distinct language disorders. (a) An unbiased ChIP screen in human neuron-like cells identified a FOXP2-bound fragment which mapped within intron 1 of the CNTNAP2 gene [21]. Locations of FOXP2 on 7q31 and CNTNAP2 on 7q35 are shown. The genomic organization of CNTNAP2, spanning $>2 \mathrm{Mb}$, is given below. Diamonds denote sites of exons, the square shows the FOXP2-bound region, and the red arrow indicates direction of transcription. Biologically independent ChIP-PCR experiments showed consistent enrichment of this region, and electrophoretic mobility shift assays (EMSAs) demonstrated strong specific binding of FOXP2. (b) mRNA expression was then assessed via quantitative RT-PCR in human neuron-like cells stably transfected with FOXP2 or with an empty control vector [21]. Levels of CNTNAP2 mRNA were inversely proportional to that of FOXP2. Findings were consistent for three sets of primer pairs (primers A-C) recognizing distinct combinations of CNTNAP2 exons. Expression changes are given as mean log2 ratios in FOXP2-positive cells compared with empty controls, normalized for equal expression of the GAPDH control; $p$-values were calculated using two-tailed unpaired $t$ tests $\left(* * * *=p<0.0001,{ }^{* * *}=p<0.001\right)$. (c) Next, SNPs from the CNTNAP2 locus were tested for association with language deficits in 184 families affected with typical SLI, using quantitative transmission disequilibrium testing (QTDT) [21]. Nine SNPs in the region between exons 13-15 showed significant evidence of association with deficits in NWR (nonsense-word repetition), a powerful endophenotype for SLI (peak association, $\mathrm{P}=0.00005$ at $\mathrm{rs} 17236239 ; \mathrm{P}=0.0019$ after multiple-testing adjustment). A putative risk haplotype ( $h t 1)$ was identified incorporating these nine SNPs. When children were divided into three groups based on the numbers of copies of $h t 1$ that they carried, it was found that mean NWR dropped by $\sim 6$ points ( 0.4SDs) as a consequence of carrying $>0$ risk alleles. Exon 13-15 SNPs have also been implicated in language delays in autism [27]. Error bars represent standard errors. Figure adapted, in part, from Ref. [21].

repetitive behaviours [27-29]. One study identified an association between delayed language in autistic children and SNPs in the same exon 13-15 region as that implicated by the SLI study [27]. Although children with autism were excluded from the SLI sample of Vernes et al. [21], a subset of SNPs were commonly associated in both studies, with the same alleles conferring risk [21,27]. Thus, altered CNTNAP2 function and/or regulation might be a shared mechanism mediating language-related deficits in distinct disorders.

\section{Functional genomics of FOXP2}

Beyond CNTNAP2, high-throughput analyses of promoter occupancy indicate that FOXP2, like other transcription factors, could regulate many downstream genes (Figure 2). Two independent 'ChIP-chip' studies coupled FOXP2ChIP to screening of microarrays containing $\sim 5000$ human promoters; one employed neuronal-like cell lines [30], the other investigated human fetal brain and lung tissue [31]. Each study uncovered several hundred potential targets. Despite use of antibodies recognizing different FOXP2 epitopes, there were significant overlaps in lists of highly enriched promoters, and these were found to contain an excess of sequences matching FOXP2-binding motifs $[30,31]$. Gene-Ontology analyses of the putative targets indicated roles in CNS patterning, development and function, including signal transduction, neurite outgrowth and axon guidance, neurotransmission and synaptic plasticity. Subsets of isolated targets were further investigated using RT-PCR in cellular models and, in a few cases, in vivo in mutant mice. This provided proof of principle of FOXP2 regulation, confirming that the protein usually represses transcription [30,31]. Additional studies are needed to determine which ChIP-chip targets are most relevant for mediating effects of FOXP2 on speech and language. Thus far, CNTNAP2 is the only known FOXP2 target tested in people with language impairment [21].

These FOXP2 ChIP-chip screens queried a small percentage of human genes and were limited to well-characterized promoters [30,31]. More comprehensive large-scale FOXP2-ChIP investigations could provide substantial new insights, especially because cis regulatory sequences often lie outside classically defined promoter regions. The work highlights another important feature of transcription-factor biology: occupied genomic regions and regulated targets often differ from one cell-type to the next [31]. For example, in their FOXP2 ChIP-chip study, Spiteri et al. [31] assessed fetal lung tissue and two relevant regions of human fetal brain, the developmental precursors of the inferior frontal cortex (IFC) and BG. These regions form part of a distributed network supporting language perception and production [32]. The left IFC contains Broca's area, classically framed as a key neural substrate for speech production, but now known to have broader roles, 


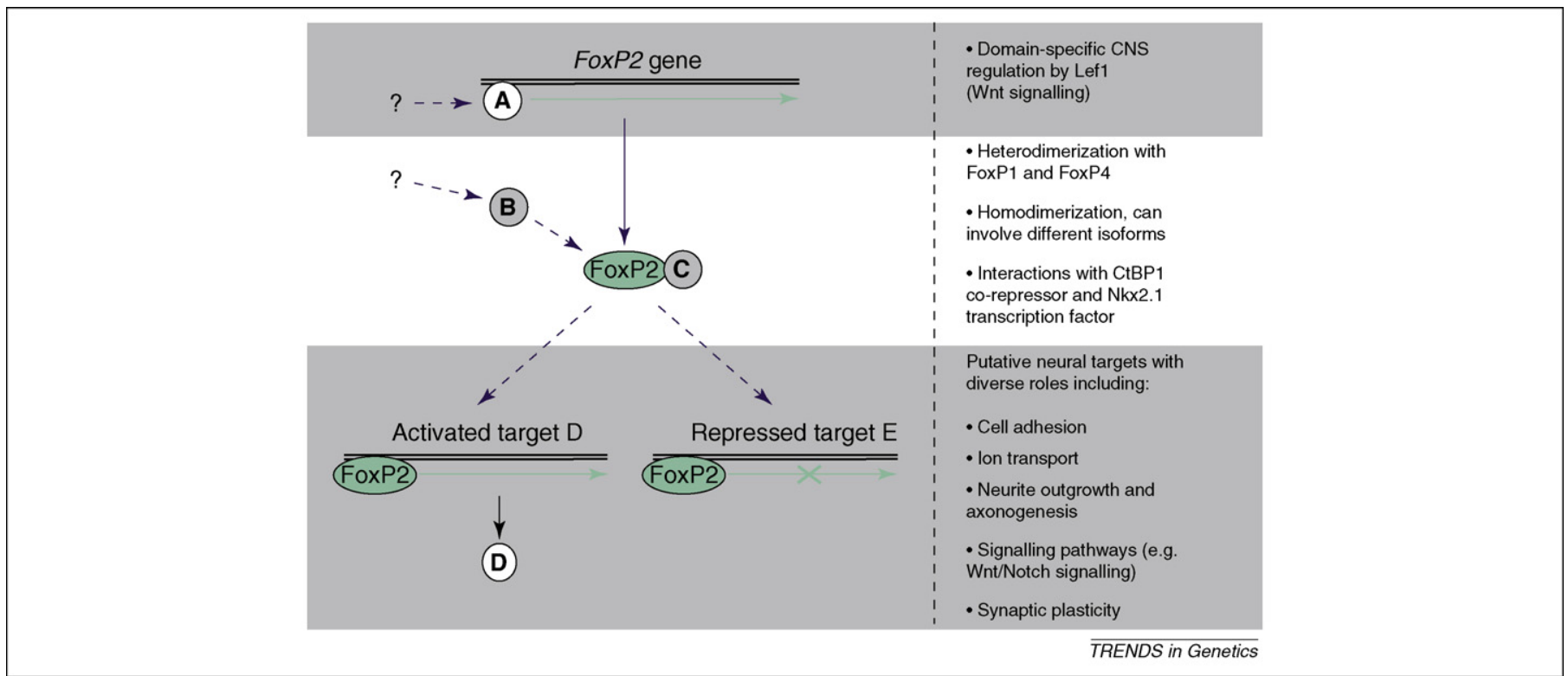

Figure 2. The FoxP2 transcription factor as an entry point into functional genetic pathways. Model systems and functional genomics are being employed to search for key factors at multiple levels, including those which act upstream of the FoxP2 gene to regulate its expression A, signalling pathways which might modify the intracellular localization or activity of the encoded protein B, other proteins with which it interacts $C$, and downstream targets which it directly activates D or represses $E$. Note, here we are using the general symbol FoxP2, because we refer to findings from different species. The right hand side of the figure gives examples of how the various levels are being dissected. Upper panel: zebrafish studies identified enhancers in the FoxP2 genomic locus which are bound by Lef1 (a member of a family of transcription factors activated by Wnt signalling) helping to drive FoxP2 expression in the tectum, mid-hindbrain boundary and hindbrain during CNS development [69]. Work in human celllines and tissue has identified multiple transcription start sites of FOXP2 with varying cellular specificities [70]. Middle panel: studies in humans, mice and songbirds indicate that there are several CNS sites at which FoxP2 expression overlaps with that of other members of the FoxP subfamily [11,13]. Functional experiments show that the different FoxP proteins heterodimerize via a highly conserved leucine-zipper and zinc-finger region [71]. These observations suggested FOXP1 as a candidate susceptibility factor in speech and language impairments [13], but screening efforts have not found mutations causing developmental verbal dyspraxia [72]. FoxP2 also homodimerizes, which might enable regulation of activity via distinct isoforms of the protein generated by alternative splicing, including one that lacks a forkhead domain [40]. It has been shown that FoxP2 interacts with the CtBP1 co-repressor [71] and the homeodomain transcription factor Nkx2.1 in the lung [73]; it is not known whether such interactions are important in the CNS. Lower panel: ChIP experiments for human FOXP2 enabled identification of direct targets in the developing brain and in neuronal cell models, and revealed that the protein usually acts as a repressor [30,31]. Putative targets come from a range of GO categories, and include several interesting classes related to neural development, patterning and function. No full genome-wide screen for direct targets has yet been reported, so many more remain to be discovered, and the profile could vary from one neuronal subpopulation to another.

including involvement in language comprehension and motor control beyond speech. Likewise, the BG are implicated in both motor and sensory aspects of language function [32]. Some putative targets were identified by ChIPchip commonly in IFC, BG and lung, and others were detected in two out of the three, whereas most were only isolated in one region. However, the latter does not necessarily indicate tissue-specificity for the FOXP2-target interaction, because only three tissues and/or regions were assessed. Moreover, final target lists for ChIP-chip are derived by imposing an artificial threshold for ChIPenrichment on quantitative data from all assessed promoters; conceivably, a true shared target might exceed the threshold in one tissue sample but not quite meet it in another, and be mischaracterized as 'specific'. Given the complexities of FOXP2 expression patterns, involving diverse neuronal subtypes in distributed circuits [10-17], a future challenge is to define neuron-specific profiles of transcriptional targets, and relate these to neural function.

\section{Animal models}

Many open questions about how FOXP2 mutations disturb speech and language development cannot be answered by studying humans. Can they be addressed in other species? Human language requires multiple processes with different levels of complexity; clearly no animal model can adequately mirror all of them. Nevertheless, certain aspects are amenable for investigation in non-human species. Spoken language depends on motor control of vocal output and auditory processing of speech signals. In species that learn complex vocalizations through imitation (e.g. humans, three orders of birds, ocean mammals, bats and possibly elephants) [19], auditory processing and motor control must integrate. The relevant neural underpinnings can be assessed in animals and could be similar in humans. But what of the essence of language: grammar, abstraction, meaning, thoughts? There is no evidence so far that animals that communicate vocally use language-like grammar systems to refer to objects or actions. However, it is an immense challenge to search for rule systems in complex streams of vocalizations in animals with large sound repertoires; this kind of effort has yet to be carried out on an adequate scale. Even if such rules were deciphered, we would still have the problem put forward by Wittgenstein, 'If a lion could speak, we would not understand him'.

Where does this leave us with respect to FoxP2 function? Brain imaging studies of affected members of the $\mathrm{KE}$ family revealed distributed structural and functional anomalies in regions that overlap with FOXP2 expression sites, including the IFC and the BG [33-35]. Given similarities in neural expression across species [10-17], animal models can help address whether abnormalities of this nature relate to roles of the gene during fetal brain development, and/or to postnatal dysfunction of differentiated neural circuits [20]. Mouse models enable sophisti- 
cated investigations of cell proliferation, cell-fate choice, neuronal migration, differentiation, formation of neural circuits, synaptic function and so on. Investigating the impacts of FoxP2 on animal vocalizations is another avenue to pursue. Is the gene important for the function of sensory, motor, or sensory-motor integration sites in the brain? Might learning to imitate sounds require it? What features of vocalizations are affected by lack of FoxP2? These are the kinds of questions that can be addressed effectively in animal models.

\section{Foxp2 in murine development}

Several mouse models have been developed in which Foxp 2 is disrupted. These models include a targeted knockout (Foxp2-KO) [36], a conditional null enabling constitutive or selective inactivation (Foxp2-flox) [37], an allelic series generated by ENU mutagenesis (R552H-ENU, S321X$E N U, N 549 K-E N U$ ) [38], and a targeted knock-in $(R 552 H-K I)$ [39]. The R552H models (R552H-ENU, R552H-KI) carry a Foxp2 substitution identical to the human R553H substitution of the KE family, whereas $S 321 X-E N U$ harbours a premature stop codon close to the human R328X nonsense mutation and seems to function as a null (Figure 3).

Pups with homozygous Foxp2 disruptions are developmentally delayed with severe motor impairments, dying $\sim 3-4$ weeks after birth [36-39]. Such findings are consistent for diverse Foxp 2 knockouts and mutants, including both $\mathrm{R} 552 \mathrm{H}$ models, in line with the Foxp2-R552H substitution (and its human equivalent FOXP2-R553H [40]) producing a loss-of-function. N549K-ENU homozygotes show milder phenotypes with longer survival, owing to a conservative arginine-to-lysine substitution in the forkhead domain [38]. Foxp2 is highly expressed in the developing lung epithelium [41]. Thorough evaluation of lungs of Foxp2-KO mice identified subtle but significant postnatal dilation of distal airspaces in homozygotes, albeit without detectable anomalies in number or morphology of epithelial cells [41]. It has been suggested that these observations might explain postnatal lethality of homozygotes [41], but such a hypothesis awaits formal testing, for example by using conditional alleles [37] for lung-specific Foxp2 disruption.

The brains of Foxp2 mouse models seem grossly normal on histological analyses [36-39] except for the cerebellum of homozygotes, which is disproportionately small with reduced foliation [37-39]. Cerebellar Foxp2 expression in wild-type animals is confined to Purkinje cells (PCs) and deep nuclei $[10,11]$. PCs influence granule cell precursor proliferation, partly by releasing Sonic hedgehog, a process driving cerebellar growth and foliation in the first two postnatal weeks [42]. Although one investigation of the cerebellum in homozygotes described ectopically placed $\mathrm{PCs}$ and abnormal retainment of an external granular layer (EGL) at postnatal day (P)15-17 [36], other studies reported intact cerebellar histoarchitecture at P19-21, with normally aligned PCs and no persistent EGL [3739]. In some cases, PCs of homozygotes had less elaborate dendritic arbors with reduced synaptophysin reactivity $[36,39]$. Apparently, complete absence of functional Foxp2 impairs postnatal cerebellar maturation, without affecting the gross morphology of other Foxp2 expression sites in the brain (although subtle developmental anomalies might have escaped detection). However, the mechanisms mediating these effects are unknown.

There are no examples of homozygous FOXP2 mutation in humans; all reports of FOXP2-related speech and language disorder are heterozygous or hemizygous [4,5,43-46]. Thus, heterozygote animal models could prove most informative for identifying relevant neural mechanisms. However, phenotypic descriptions of heterozygous Foxp2 mouse models vary. Shu et al. [36] reported developmental and motor delays in Foxp2-KO heterozygotes, intermediate in strength between homozygotes and wild types. French and colleagues saw no such abnormalities in heterozygous nulls (generated via constitutive Cre-based inactivation of a single Foxp2-flox allele) despite a similar half-dosage of Foxp2 protein [37]. Groszer et al. [38] also observed normal postnatal development in all heterozygotes of their allelic series (R552H-ENU, S321X-ENU, N549K-ENU). Finally, Fujita and colleagues reported a small mean weight reduction $(\sim 10-15 \%)$ for $R 552 H-K I$ heterozygotes, but this effect could be attributable to the $<2 \%$ outliers with severely reduced weight and motor inactivity; most $R 552 \mathrm{H}$-KI heterozygotes seemed normal [39]. Cerebellar morphology of heterozygotes in these different studies ranged from moderately abnormal [36] or developmentally delayed [39] to grossly normal [37,38].

Conflicting heterozygous data are not accounted for by the nature of Foxp2 mutation. French et al. [37] and Groszer et al. [38] assessed four different alleles; all heterozygotes developed normally. Perhaps factors in the genomic background modulate effects of partially reduced Foxp2 dosage. Whereas French and colleagues and Groszer and colleagues characterized animals crossed onto pure C57Bl/6 or C3H backgrounds [37,38], Shu et al. [36] and Fujita et al. [39] assessed F1 heterozygotes on a mixed (C57Bl/6x129) background.

\section{Foxp2 and mouse vocalization}

The vocal repertoire of laboratory rodents includes frequency-modulated sonic $(\sim 20-20000 \mathrm{~Hz})$ and ultrasonic $(>20 \mathrm{KHz})$ sounds. Sonic vocalizations are produced by vibrating vocal cords, whereas ultrasounds depend on expiration of air through tightly opposed nonvibrating vocal cords. They are sometimes preceded by emission of a broadband clicking sound [47]. Young mouse pups produce innately specified calls automatically in response to altered arousal [47]. Adult male mice, on encountering female mice or pheromones, produce ultrasonic 'songs' comprising structured sequences that contain several syllable types [48]. However, it remains unknown whether these adult 'songs' constitute learned vocalizations, and the underlying neurobiology has yet to be characterized.

Thus far, studies of Foxp2 in mouse vocalization have focused only on innate pup calls [36,38,39]. On isolation from the mother and/or nest, pups emit ultrasounds that elicit retrieval by the parent; this is similar to the response of human adults to a crying baby [47]. Investigations of Foxp2-KO, R552H-ENU and R552H-KI mice concur that homozygotes lacking functional Foxp2 do not produce ultrasonic isolation calls $[36,38,39]$. Although Foxp2-KO 
(a)

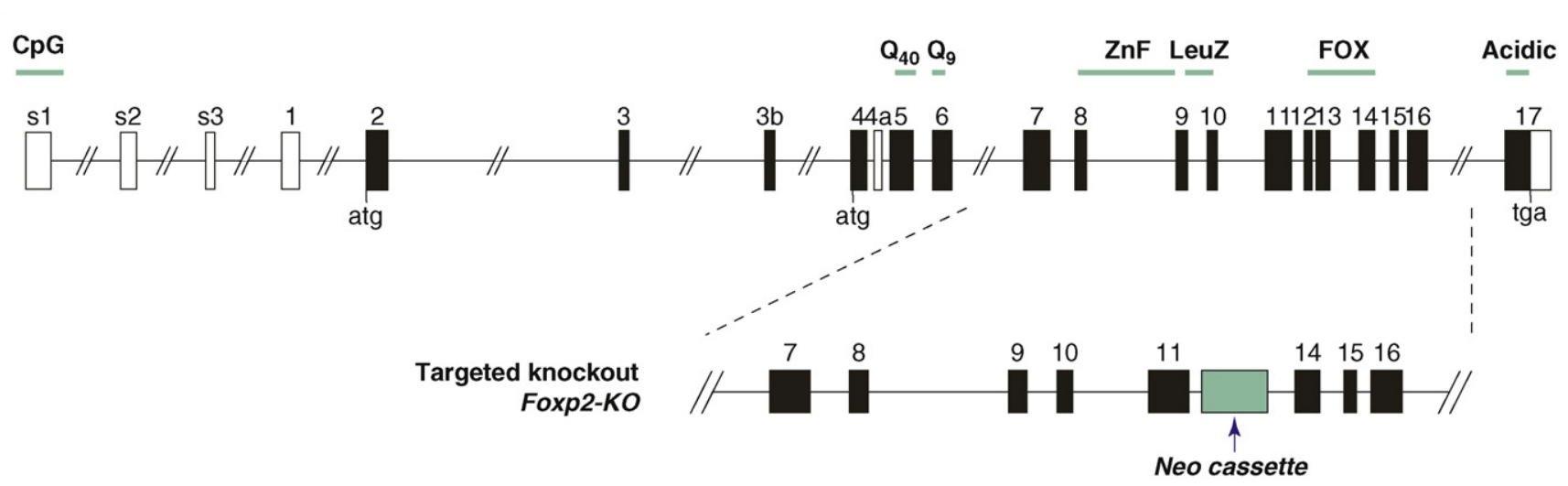

Conditional null allele

Foxp2-flox

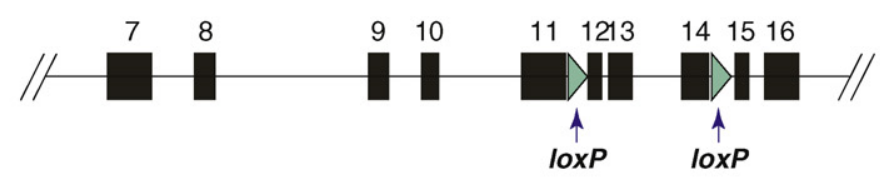

Allelic series of ENU mutations S32IX-ENU, N549K-ENU, R552H-ENU

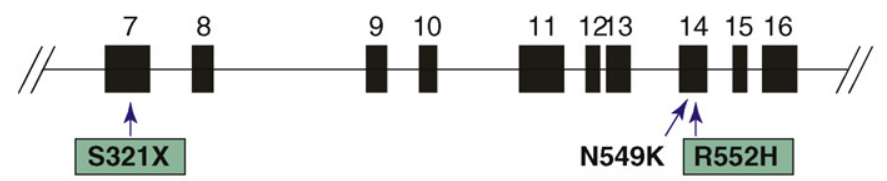

Targeted R552H knockin

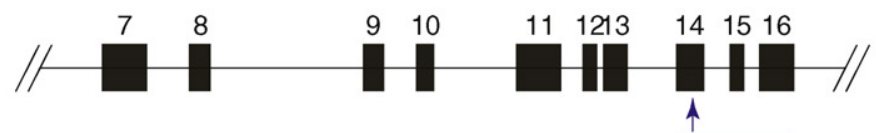

R552H

(b)

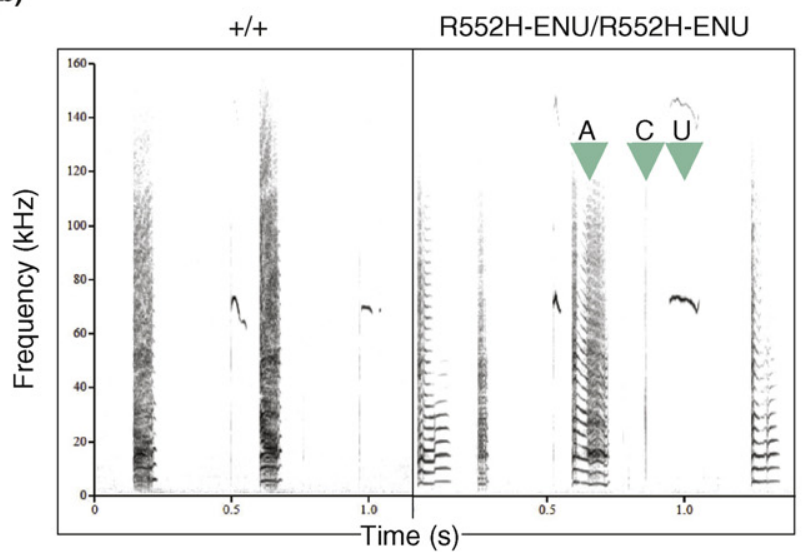

(c)

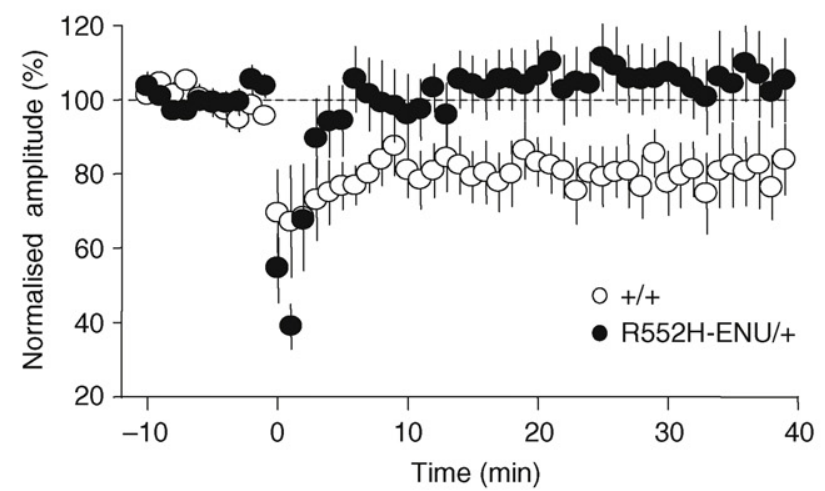

TRENDS in Genetic

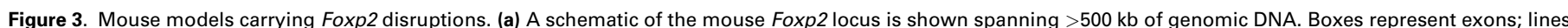

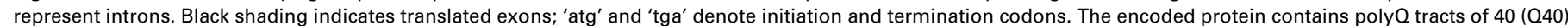

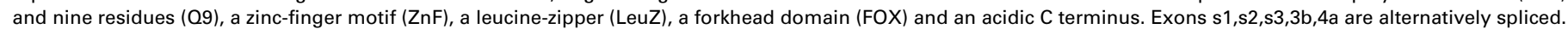

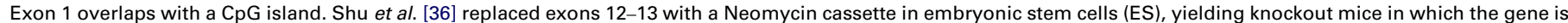

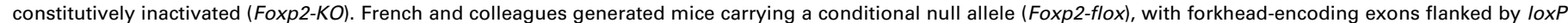



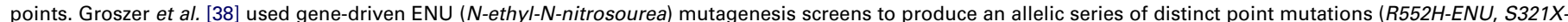

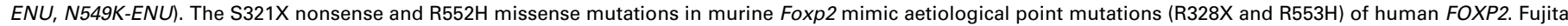

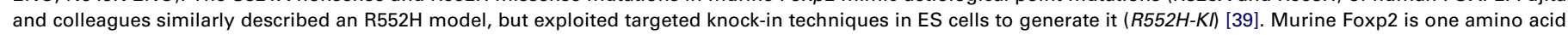



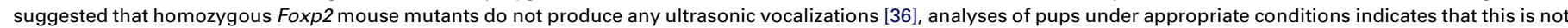

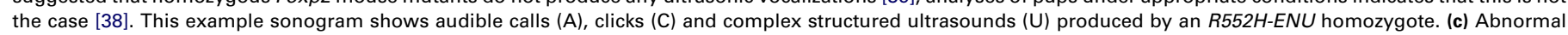

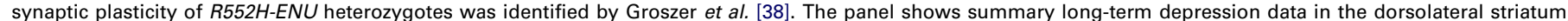


heterozygous mutants do not. Parts (b) and (c) adapted from Ref. [38]. 
and $R 552 \mathrm{H}-\mathrm{KI}$ mice were only studied during isolation, $R 552 H-E N U$ vocalizations have also been analysed in situations involving greater arousal [38]. When pups are lifted above the ground with gentle pressure on the tail, they emit audible distress calls with interspersed ultrasounds and clicks. In this context, R552H-ENU homozygotes, like wild types, produced all three types: audible calls, ultrasounds and clicks [38]. Thus, contrary to initial reports [36], Foxp2 is not essential for ultrasonic vocalization in mice - homozygotes generate harmonically structured audible calls and complex ultrasounds, but only under elevated stress (Figure 3). R552H-ENU homozygotes emitted the same total number of vocalizations in distress as did littermate controls, although these comprised more clicks and fewer ultrasounds, and the latter had lower sound-pressure levels with shorter durations [38]. Crucially, mice with homozygous Foxp2 disruptions also have substantial developmental delays, general motor impairment, reduced spontaneous activity and increased dilation of distal lung airspaces. Thus, their vocalization difficulties could be secondary to other problems.

Impacts of arousal state and developmental delay are again relevant for heterozygote vocalizations. Shu et al. [36] reported that Foxp2-KO heterozygotes emitted significantly fewer ultrasonic isolation calls than wild types, with unaltered call properties (duration, peak frequency, bandwidth). As noted earlier, Foxp2-KO heterozygotes developed more slowly than wild types. These delays constitute an important confounding factor; rates of ultrasound emission follow strain-specific ontogenetic profiles, closely related to stages of postnatal development [49]. Fujita et al. [39] also reported developmental delays and reduced ultrasound emission for $R 552 \mathrm{H}-\mathrm{KI}$ heterozygotes, although no statistical analyses were performed. Without providing formal analyses of data, this study also claimed that wild types produced just one type of vocalization, whereas heterozygotes additionally emitted shorter-length ultrasounds and clicks. By contrast, Groszer et al. [38] found that $R 552 H-E N U$ heterozygotes developed normally, and pup vocalizations (including rates of production of different call types) did not differ significantly from wildtype littermates.

In sum, relationships between Foxp2 disruption and altered mouse vocalization are less straightforward than some reports suggest $[36,39]$. We should be wary of drawing simplistic correspondences between rodent pup vocalizations and human speech. Little is known at present regarding the neural systems that control mouse ultrasound emissions, and whether there are parallels to those controlling articulatory gestures in humans. Moreover, pup vocalizations are innate and are produced at early developmental time points when the mice are still deaf [47], whereas speech involves voluntary control of learned vocalizations. Thus, there are currently no grounds for claiming (as in Ref. [39]) discovery of a common molecular mechanism mediating mouse ultrasounds and human speech learning. Nor has the work demonstrated clear and/or specific causal links between putative Foxp2mediated abnormalities in cerebellar PCs and ultrasonic vocalization deficits; this would require experiments involving PC-specific Foxp2 disruption. Additionally, no study has yet shown any impact of murine Foxp2 on social interactions or adult vocalizations.

\section{Foxp2, motor-skill learning and synaptic plasticity}

When considering FOXP2 in human speech and language, it is important to adopt a sophisticated approach, which does not assume that the gene - and orthologues in other species - must be intrinsically tied to roles in communication or production of vocalizations. Indeed, based on neuropsychological and neuroimaging investigations of the KE family, it has been proposed that the FOXP2related speech and language disorder involves core deficits in learning, planning and executing rapid movement sequences $[7,50]$. These effects are thought to be underpinned by neural abnormalities in frontostriatal and/or frontocerebellar circuitry [33-35], networks implicated in sensorimotor integration and motor-skill learning [51], which include key FOXP2 expression sites [10]. But what impact does an $\mathrm{R} 552 \mathrm{H}$ change in mouse Foxp2 (equivalent to FOXP2-R553H in the KE family) have on rapid motorskill learning?

Like other mice lacking functional Foxp2, R552H-ENU homozygotes are of limited value for behavioural studies because they have developmental delays, severe motor dysfunction and postnatal lethality. However, $\mathrm{R} 552 \mathrm{H}$ $E N U$ heterozygotes (matching the genetic status of affected KE members) develop normally; weight-gain, base-line motor abilities, spontaneous activity, anxiety levels and general cognition do not differ from wild types [38]. When Groszer et al. [38] recorded species-typical motor patterns from tilted running-wheels in the cages of mice, they found that R552H-ENU heterozygotes learned more slowly than wild-type littermates, with significant differences on multiple indices. (Mice voluntarily spend considerable time on such wheels, running in characteristic short bouts [52]; there is an initial learning phase after providing the wheel, involving steep increases in bout-length, complementary drops in bout-number and rapid improvements in running speed.) Consistent findings were also observed on accelerating rotarods, a standard paradigm for assessing rodent motor-skills [38].

On performing electrophysiological examinations of key neural Foxp2-expression sites known to be important for motor-skill learning, Groszer et al. [38] found abnormalities in synaptic plasticity - the way in which synapse sensitivity is modulated in response to prior stimulation. Cerebellar PCs of R552H-ENU heterozygotes showed significantly increased paired-pulse facilitation (a type of short-term plasticity [53]) at short interstimulus intervals, without detectable disturbances in synaptic circuitry [38]. More remarkably, despite normal synaptic organization and transmission, neurons in the dorsolateral striatum (part of the BG) of heterozygotes did not show long-term depression [38]. High-frequency stimulation of glutamatergic synapses in the striatum normally induces robust long-term depression, a form of long-term plasticity, which depends on endocannabinoid retrograde signalling and has important roles in striatal-dependent learning [54,55]. Humans carrying the equivalent mutation show abnormal functional activation of the striatum during speech- and language-related tasks [35,50]. 


\section{Box 3. Molecular evolution of FoxP2 proteins}

Far from being unique to humans, FOXP2 is one of the most highly conserved of vertebrate genes [10-17]. Nonetheless, against this background, there are indications of accelerated change in FOXP2 protein sequence during human evolution [56,57,81]. Of three amino-acid substitutions distinguishing human and murine orthologues, two (T303N, N325S) occurred on the human lineage at some point after splitting from the chimpanzee lineage some 5-6 million years ago $[56,57]$. (One of these, however, also occurred independently in carnivores [57].) The acceleration on the human lineage does not seem to reflect increased mutation rate or relaxed constraint, and has been interpreted as evidence of positive selection. Additional support for a selective sweep comes from analysing intronic sequences neighbouring the putative selected sites. Patterns of genomic diversity for these sequences in extant human populations were used to estimate that the amino-acid substitutions arose recently in human history, within the past $\sim 200000$ years [56,57,81].

Krause and colleagues independently dated the origin of these substitutions, querying FOXP2 sequence in DNA from fossilized bones of two Neanderthals [82]. Curiously, they found that both amino acid sites matched modern humans, concluding that the substitutions were already present in the common Neanderthalhuman ancestor. Neanderthals split from humans at least 300000 years ago [83], so these data are difficult to reconcile with evidence of a recent selective sweep. Suggested explanations include contamination of Neanderthal samples with human DNA (although multiple controls were included to minimize this [82]) or non-zero levels of Neanderthal-human gene flow (itself a contentious issue) [81]. Alternatively, the recent episode of selection might not have involved the amino acid substitutions, but could be related to another humanspecific FOXP2 change, perhaps affecting its regulation. Until

It is not yet known whether a shared mechanism unites functional abnormalities observed in different Foxp2expressing circuits. Selective Foxp2 disruption [37] might be necessary to definitively link impaired synaptic plasticity to behavioural deficits, and would also facilitate dissection of striatal and cerebellar contributions. One intriguing implication is that contributions of human FOXP2 to spoken language might, at least in part, be built on conserved vertebrate roles in plasticity of distributed neural circuits mediating motor-skill learning, rather than vocalization or communication per se. Indeed, it is not recently, nothing was known about the functional impact of the amino acid substitutions, subtle changes lying outside known functional domains of FOXP2. However, new data from knock-in mice carrying these substitutions indicate that they affect brain development and neural plasticity in ways that differ from knockout or mutant alleles [68]

It is worth emphasizing that because language is clearly underpinned by multifactorial influences [3], the status of a single gene in ancient DNA [82] is insufficient to resolve long-standing debates over linguistic capacities of our extinct ancestors. Regardless of uncertainty over dating and functional impact of the hominin FOXP2 changes, these are unlikely to be the sole or major driving force behind the appearance of spoken language [84]. Comparative primate genomics points to myriad molecular events that could have contributed to human origins, although pinpointing which were linked to language remains a challenge [1]. As genes upstream [69] and downstream $[21,30,31]$ of FOXP2 are identified, one interesting possibility is that other elements of the relevant networks might have also undergone selection in hominins [31].

Outside of primates, analyses of molecular evolution of FoxP2 across other vertebrate species - including vocally learning and nonlearning birds, bats and cetaceans - have not reported evidence of protein-coding changes that correlate with vocal-learning capacity $[19,85]$. In contrast to the typically high conservation of FoxP2 protein in nonhuman mammals, there is extensive sequence diversity in echolocating bats, and selective pressure apparently differs between bats with contrasting sonar systems [86]. Given the considerable demands that echolocation places on rapid sensorimotor integration and co-ordination, it is tempting to posit a functional explanation, but at present such speculation is based purely on sequence comparisons, without experimental data.

certain that the impaired motor-skill learning and/or production observed in human FOXP2-related disorder is entirely confined to the orofacial system; perhaps a subtle domain-general deficit disproportionately affects speech owing to its particular dependency on rapid precise movement sequences. It is also possible that evolutionarily ancient functions in motor-skill learning circuits were recruited and modified towards unique aspects of speech and language - studies of molecular evolution indicate that FOXP2 underwent positive selection on the lineage that led to humans [56,57] (Box 3).

\section{Box 4. Language and birdsong: similarities and differences}

Like people, many songbirds interact vocally, taking turns in exchanging vocal signals. To do so, birds and humans vary the order and arrangement of vocal units ('syllables') in a rule-governed way, resulting in a rich variety of sequences. Importantly, however, human language can create infinite messages via a finite repertoire of sounds, can refer to objects, actions and thoughts, and is governed by complex rules that crucially affect meaning. There is (as yet) no evidence that any of these features exists in bird song. Yet, other parallels exist. Like language, the song of many birds requires vocal imitation during development, is constrained by innate predispositions and guided by auditory feedback. Birdsong and speech are optimally learned during crucial developmental periods and are strongly affected by social factors. As in speech development, phonological development precedes syntactical development in birds [58].

Striking similarities also exist in how speech and birdsong are processed at the neural level. Birdsong and speech are coded in analogous neural pathways that are functionally lateralized. Physiological, anatomical and imaging studies show that the auditory system of songbirds is specialized for behaviourally relevant vocalizations and that this specialization can be learned [58]. The tuning properties of neuronal ensembles coding these specializations are beginning to be understood [87]. Likewise, birdsong neurobiology has contributed enormously to our understanding of how communication sounds are learned and controlled [58]. For instance, such research established that the same brain region can be involved in both perception and production of sounds, well before the term 'mirror neuron' was even coined in mammals [88]. This concept has recently been proven for single neurons in the songbird brain [89]. The importance of the BG for speech [32] was echoed by bird research highlighting involvement of this structure in song learning and production $[59,64]$. More widely, the finding of a 'sparse' code (i.e. only a small ensemble of neurons being active during a very short time at any particular point during song production) is influencing theories about neural control of behaviour [90].

Although there is agreement regarding their overall comparability, systematic and sustained attempts to extend the comparative analysis of human speech and birdsong to more formal aspects of their structure are slow in coming. As in spoken language, birdsong phonology depends on a collection of perceptual, productive and cognitive skills. Investigations into linguistic phenomena in birdsong promise to deliver exciting insights into the biological basis of sequentially organized learned vocal behaviours. 


\section{FoxP2 and the neurobiology of birdsong}

Songbirds possess specialized brain areas for song recognition, production and learning (the 'song system'), enabling them to learn their song by imitating a tutor [58]. After memorizing the song of a tutor, young birds produce a rambling series of sounds, which become more discrete and patterned over time, culminating in the adulttypical song. Similarities to human spoken language make songbirds a popular model for studying vocal learning, although it is important to recognize the limits of these parallels (Box 4). So, is avian FoxP2 a key player in the neurobiology of birdsong?

Genes involved in vocal learning could influence formation and/or subsequent function of the relevant neural circuits. Consistent with the former notion, FoxP2 is expressed in regions of the avian embryo in which inductive signals organize adjacent neuroepithelium and neuronal migration [12]. However, neural expression persists throughout life, implying ongoing postnatal functions. As in other vertebrate species that have been investigated, birds strongly express FoxP2 in the striatum of the BG, nuclei of the dorsal thalamus and midbrain, and the inferior olive, which gives rise to climbing fibres that innervate cerebellar PCs, also FoxP2 positive [10-17]. Studies of birds and mice indicate little or no expression in cranial motor nuclei [12,17], arguing against a role in the purely peripheral control of orofacial musculature (to our knowledge no-one has yet assessed expression levels in human cranial motor nuclei). Moreover, avian Foxp2 levels are very low in motor pathways associated with innate vocalizations and song production, whereas higher levels are observed in Area X, a BG nucleus essential for song development [59]. Interestingly, in young zebra finches, Area X shows slight but

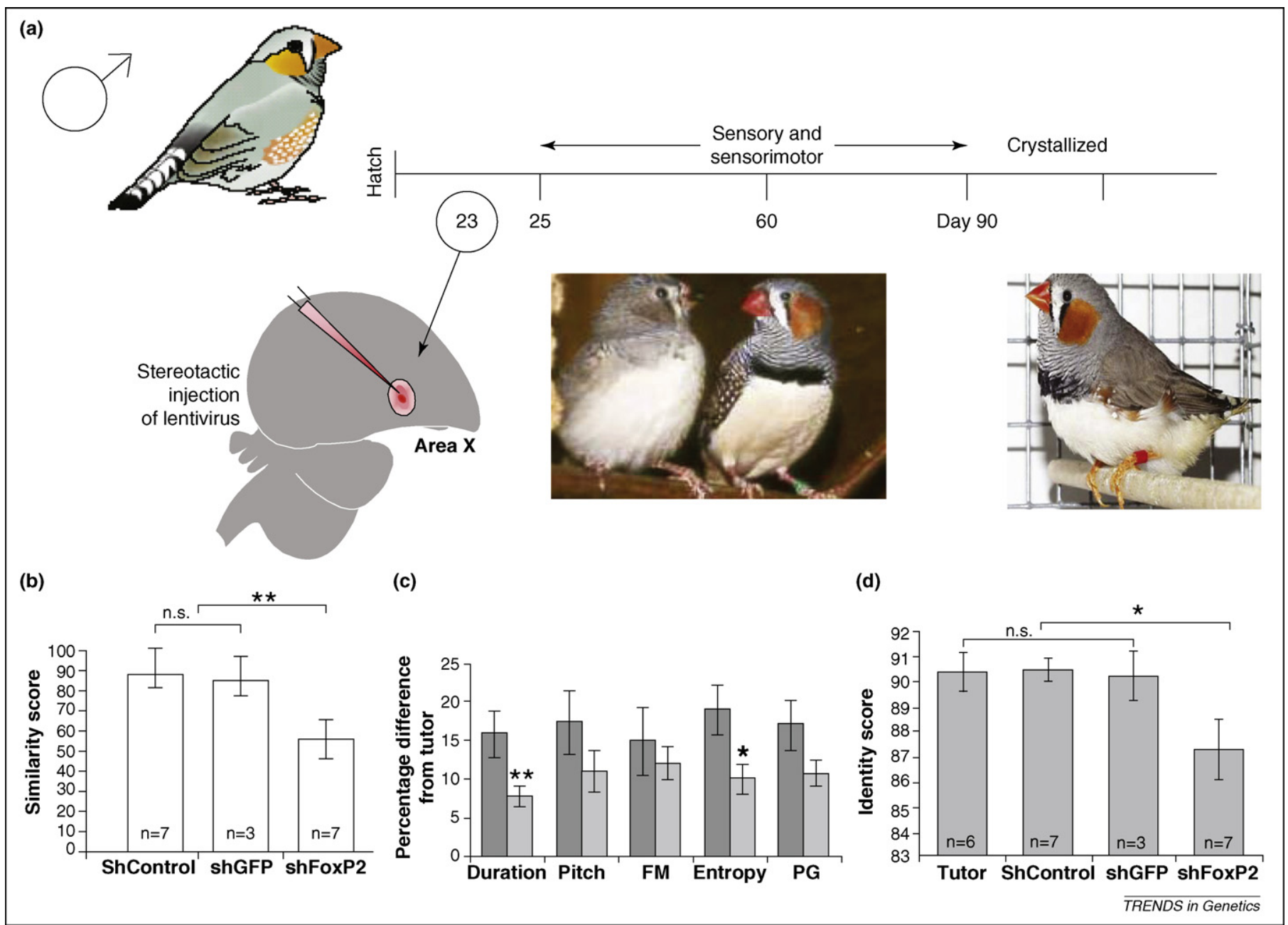

Figure 4. Incomplete and inaccurate vocal imitation by FoxP2 knockdown zebra-finch pupils. (a) Schematic of experimental design. On posthatch day (PHD) 23, at the onset of the sensory-motor learning phase FoxP2 knockdown or control viruses (shControl and shGFP) were injected stereotaxically into Area X to achieve spatial control of knockdown. Starting on PHD30, each young bird, here called pupil, was kept in a sound isolation chamber, together with an adult male zebra finch as tutor. Songs were recording during development and after reaching adulthood at approximately three months for subsequent song analyses. (b) FoxP2 knockdown pupils copy fewer syllables; the mean similarity between tutor and pupil motifs was significantly lower in shFoxP2-injected pupils than in shControl- and shGFP-injected pupils ( \pm SEM; twotailed Mann-Whitney U test, ${ }^{* *} \mathrm{p}<0.001$, Bonferroni-corrected $\alpha$-level). There was no significant difference between shGFP-and shControl-injected animals (not significant [n.s.], p > 0.5). (c) Copied syllables are less accurately imitated. Comparison of syllable duration and mean acoustic feature values between pupil syllables and their respective tutor syllables. Divergence of imitated syllables from the tutor model tended to be larger for all acoustic measures in the FoxP2 knockdown pupils (dark grey bars) than in the controls (light grey bars). For average syllable duration and mean entropy measures, the difference was significant ( \pm SEM; two-tailed Mann-Whitney $\mathrm{U}$ test, ${ }^{* *} \mathrm{p}<0.001$ for duration and ${ }^{*} \mathrm{p}<0.05$ for entropy; Bonferroni-corrected $\alpha$-level; shFoxP2 $\mathrm{n}=7$ animals, on average three syllables per animal; shControl $\mathrm{n}=7$ animals, on average four syllables per animal). (d) Acoustic variability of syllables from rendition to rendition was higher in shFoxP2-injected than in control pupils, as indicated by significantly lower syllable identity scores (two-tailed Mann-Whitney $U$ test, ${ }^{*} p<0.05$; Bonferroni-corrected $\alpha$-level). Control and tutor birds sang with comparable


$\mathrm{n}=3$, on average five syllables per animal). Figure adapted from Ref. [64]. Abbreviations: FM, frequency modulation; PG, pitch goodness. 
consistent elevation of FoxP2 levels at the time when they learn song [12]. Similarly, FoxP2 levels are elevated in Area $\mathrm{X}$ in a strain of adult canaries that remodel their songs seasonally during late summer and fall, at the end of the breeding season [12]. Thus, developmental and seasonal changes in Area-X FoxP2 expression correlate with phases of vocal plasticity.

These effects do not seem to be a reflection of songdriven gene-regulation (e.g. as seen for zenk [60]) because the birds under study had not sung in the hours before analysis. Nevertheless, it seems that postnatal FoxP2 expression can vary with prior singing activity. In Area $\mathrm{X}$ of adult zebra finches, FoxP2 mRNA levels are on average lower when a male sings by himself than after singing courtship-song to a female [61]. A western blotting study observed reduced Area-X FoxP2 protein levels two hours after singing in either condition [62], when normalized against a GAPDH protein control. Unfortunately, this investigation did not determine whether GAPDH expression in Area X is indeed unaltered by song - in fact, GAPDH levels in neurons that project from the pallial premotor song-nucleus HVC towards Area X are reduced by $>6$ times 90 min after singing [63].

A recent study assessed causal links between FoxP2 expression and vocal plasticity, using lentivirus-mediated RNA-interference to experimentally reduce FoxP2 levels in Area $\mathrm{X}$ in vivo [64] (Figure 4). Before the onset of song learning, FoxP2-targetting lentiviral constructs or control viruses were stereotaxically injected into Area X of juvenile zebra finches, yielding successful FoxP2 knockdown. Expression levels were typically reduced by 50\% [64], mirroring the reduced dosage of humans with FOXP2related speech and language disorder (Box 1). After tutoring by adult males, songs of knockdown and control 'pupils' were analysed, once they had reached adulthood. Remarkably, FoxP2 knockdown birds imitated tutor songs incompletely, copying some notes but omitting others. Notes that were imitated were copied less accurately than in controls, and production of syllables varied more from song to song [64]. Such findings are intriguing in light of the incomplete and inaccurate renditions of words and highly variable pronunciation observed in humans carrying FOXP2 mutations. In finches, the phenotype might be due to impaired sensory-motor integration, particularly when birds adjust their songs through vocal motor practice. Further experiments are needed to distinguish this from explanations in terms of motor execution or sensory deficits. It is unlikely that Area-X FoxP2 knockdown yields purely motoric deficits. Syllables that were not imitated by knockdown pupils did not differ in acoustic features from syllables that were imitated. Likewise, analyses across the entire syllable repertoire indicated that all zebra-finchtypical syllable features could be produced by knockdown birds, but that their imitation was impaired [64].

How might Area-X FoxP2 expression influence vocal variability and song imitation? Spiny neurons in this nucleus receive pallial glutamatergic input from neurons in the HVC [65] which are active during singing and during auditory stimulation [66]. FoxP2-expressing spiny neurons also receive midbrain dopaminergic input [12]. In mammals, nigral dopamine acts on various behavioural sys- tems, including reward learning [67]. The integration of pallial and dopaminergic signals in FoxP2-expressing spiny neurons represents a plausible 'hub' for adjusting song motor output to the tutor song memory during learning. Increased FoxP2 expression at times of vocal plasticity might mediate adaptive structural and functional changes of these spiny neurons, affecting motor learning through up- or down-regulation of neural plasticity-related genes.

\section{The future of FOXP2}

The research described here, covering just a subset of tantalizing findings from FOXP2 studies, indicates the extraordinary breadth of topics opened up by the discovery of this gene. Beginning with a rare monogenic syndrome with only limited clinical impact, the recent investigations touch on areas across multiple fields and in diverse species; aetiology of common human disorders, impact of transcription factors on nervous system development, neural mechanisms involved in motor-skill learning and vocalization, even extending to fundamental questions about hominin evolution. One recurrent theme at the core of the expanding literature on FOXP2 function is its potential role(s) in neural plasticity of distributed circuits in the vertebrate brain. Reduced functional FoxP2 protein seems to yield speech and language deficits in humans, impaired vocal learning in songbirds, and abnormal synaptic plasticity and motor-skill learning deficits in mice. It remains to be seen if consistent molecular mechanisms underlie these observations; one attractive hypothesis is that the protein

\section{Box 5. Outstanding questions}

- How do upstream factors regulate expression of FoxP2 in different circuits of the vertebrate brain? What are the species-specific differences in regulation of expression and are they evolutionarily important?

- What are the specific functional risk variants in the exon 13-15 region of CNTNAP2 that account for the association with nonword repetition deficits in SLI and how do they affect regulation or function of this gene? Are language delays in autism associated with the same functional variants, as might be suggested by the SNP data? Do common variants at the CNTNAP2 or FOXP2 loci have any association with variability in language performance in the normal range in the general population?

- What is the full range of neural targets regulated by FOXP2? How does homodimerization and heterodimerization with other FOXP proteins affect target regulation? How does the profile of regulated targets vary from one neuronal subtype to another, and/or at different developmental time points? Does downstream target regulation differ among species? Which FOXP2 targets are most relevant for explaining speech and language disorders in people carrying mutations? Are any others, in addition to CNTNAP2, associated with impairments in common forms of SLI?

- Does reduced functional dosage of Foxp2 in rodents affect features of adult ultrasonic 'songs'? Does learning have a role in rodent vocalizations? How are they controlled neurally?

- What is the underlying molecular mechanism that accounts for altered synaptic plasticity in mice with Foxp2 mutations? How do synaptic plasticity anomalies relate to changes at the behavioural level (e.g. in motor-skill learning)? Are plasticity deficits confined to only a subset of Foxp2-expressing circuits?

- Overall, how do roles of FoxP2 differ in different neuronal subpopulations and circuits at distinct developmental time points and in the mature organism?

- Can in vivo knockdown of FoxP2, as used in songbirds, be applied to other vocal learning species? 
normally acts to modulate neural plasticity in relevant circuits by repressing genes that are typically induced by neuronal activity. Continued analyses of the crucial gene regulatory networks (upstream and downstream pathways) are underway, along with further electrophysiological characterization of FoxP2-expressing neurons in animal systems (Box 5). Moreover, a new study engineered mice to carry the two amino acid substitutions that occurred during human evolution (Box 3) and observed that medium spiny neurons in the BG showed increased dendritic length and increased synaptic plasticity [68], contrasting with effects associated with reduced Foxp2 dosage [38]. These and other such efforts will not only shed new light on FoxP2 function in the vertebrate brain, but might also illuminate some of the molecular and cellular foundations on which human spoken language is built.

\section{Acknowledgements}

Research in the laboratory of C.S. is supported by the DFG Sonderforschungsbereich 665 and the Max Planck Society. Research in the laboratory of S.E.F is supported by the Royal Society, the Wellcome Trust and Autism Speaks. S.E.F is a Royal Society Research Fellow.

\section{References}

1 Fisher, S.E. and Marcus, G.F. (2006) The eloquent ape: genes, brains and the evolution of language. Nat. Rev. Genet. 7, 9-20

2 Fisher, S.E. and Francks, C. (2006) Genes, cognition and dyslexia: learning to read the genome. Trends Cogn. Sci. 10, 250-257

3 Fisher, S.E. et al. (2003) Deciphering the genetic basis of speech and language disorders. Annu. Rev. Neurosci. 26, 57-80

4 Lai, C.S. et al. (2001) A forkhead-domain gene is mutated in a severe speech and language disorder. Nature 413, 519-523

5 MacDermot, K.D. et al. (2005) Identification of FOXP2 truncation as a novel cause of developmental speech and language deficits. Am. J. Hum. Genet. 76, 1074-1080

6 Bishop, D.V. (2001) Genetic and environmental risks for specific language impairment in children. Philos. Trans. R. Soc. Lond. B Biol. Sci. 356, 369-380

7 Watkins, K.E. et al. (2002) Behavioural analysis of an inherited speech and language disorder: comparison with acquired aphasia. Brain 125, $452-464$

8 Fisher, S.E. (2007) Molecular windows into speech and language disorders. Folia Phoniatr. Logop. 59, 130-140

9 Cirillo, L.A. and Barton, M.C. (2008) Many forkheads in the road to regulation. Symposium on forkhead transcription factor networks in development, signalling and disease. EMBO Rep. 9, 721-724

10 Lai, C.S. et al. (2003) FOXP2 expression during brain development coincides with adult sites of pathology in a severe speech and language disorder. Brain 126, 2455-2462

11 Ferland, R.J. et al. (2003) Characterization of Foxp2 and Foxp1 mRNA and protein in the developing and mature brain. J. Comp. Neurol. 460, 266-279

12 Haesler, S. et al. (2004) FoxP2 expression in avian vocal learners and non-learners. J. Neurosci. 24, 3164-3175

13 Teramitsu, I. et al. (2004) Parallel FoxP1 and FoxP2 expression in songbird and human brain predicts functional interaction. J. Neurosci. $24,3152-3163$

14 Bonkowsky, J.L. and Chien, C.B. (2005) Molecular cloning and developmental expression of foxP2 in zebrafish. Dev. Dyn. 234, 740-746

15 Shah, R. et al. (2006) Expression of FoxP2 during zebrafish development and in the adult brain. Int. J. Dev. Biol. 50, 435-438

16 Takahashi, K. et al. (2008) Expression of FOXP2 in the developing monkey forebrain: comparison with the expression of the genes FOXP1, PBX3, and MEIS2. J. Comp. Neurol. 509, 180-189

17 Campbell, P. et al. (2009) Conservation and diversity of Foxp2 expression in muroid rodents: functional implications. J. Comp. Neurol. 512, 84-100
18 Hauser, M.D. and Bever, T. (2008) Behavior. A biolinguistic agenda. Science 322, 1057-1059

19 Scharff, C. and Haesler, S. (2005) An evolutionary perspective on FoxP2: strictly for the birds? Curr. Opin. Neurobiol. 15, 694-703

20 White, S.A. et al. (2006) Singing mice, songbirds, and more: models for FOXP2 function and dysfunction in human speech and language. $J$. Neurosci. 26, 10376-10379

21 Vernes, S.C. et al. (2008) A functional genetic link between distinct developmental language disorders. N. Engl. J. Med. 359, 2337-2345

22 Inda, M.C. et al. (2006) Voltage-gated ion channels in the axon initial segment of human cortical pyramidal cells and their relationship with chandelier cells. Proc. Natl. Acad. Sci. U. S. A. 103, 2920-2925

23 Abrahams, B.S. et al. (2007) Genome-wide analyses of human perisylvian cerebral cortical patterning. Proc. Natl. Acad. Sci. U. S. A. $104,17849-17854$

24 Strauss, K.A. et al. (2006) Recessive symptomatic focal epilepsy and mutant contactin-associated protein-like 2. N. Engl. J. Med. 354, 13701377

25 The SLI Consortium (2002) A genomewide scan identifies two novel loci involved in specific language impairment. Am. J. Hum. Genet. 70, 384398

26 The SLI Consortium (2004) Highly significant linkage to the SLI1 locus in an expanded sample of individuals affected by specific language impairment. Am. J. Hum. Genet. 74, 1225-1238

27 Alarcon, M. et al. (2008) Linkage, association, and gene-expression analyses identify CNTNAP2 as an autism-susceptibility gene. Am. J. Hum. Genet. 82, 150-159

28 Arking, D.E. et al. (2008) A common genetic variant in the neurexin superfamily member CNTNAP2 increases familial risk of autism. Am. J. Hum. Genet. 82, 160-164

29 Bakkaloglu, B. et al. (2008) Molecular cytogenetic analysis and resequencing of contactin associated protein-like 2 in autism spectrum disorders. Am. J. Hum. Genet. 82, 165-173

30 Vernes, S.C. et al. (2007) High-throughput analysis of promoter occupancy reveals direct neural targets of FOXP2, a gene mutated in speech and language disorders. Am. J. Hum. Genet. 81, 1232-1250

31 Spiteri, E. et al. (2007) Identification of the transcriptional targets of FOXP2, a gene linked to speech and language, in developing human brain. Am. J. Hum. Genet. 81, 1144-1157

32 Lieberman, P. (2002) On the nature and evolution of the neural bases of human language. Am. J. Phys. Anthropol. (Suppl 35), 36-62

33 Watkins, K.E. et al. (2002) MRI analysis of an inherited speech and language disorder: structural brain abnormalities. Brain 125, 465-478

34 Belton, E. et al. (2003) Bilateral brain abnormalities associated with dominantly inherited verbal and orofacial dyspraxia. Hum. Brain Mapp. 18, 194-200

35 Liegeois, F. et al. (2003) Language fMRI abnormalities associated with FOXP2 gene mutation. Nat. Neurosci. 6, 1230-1237

$36 \mathrm{Shu}, \mathrm{W}$. et al. (2005) Altered ultrasonic vocalization in mice with a disruption in the Foxp2 gene. Proc. Natl. Acad. Sci. U. S. A. 102, 96439648

37 French, C.A. et al. (2007) Generation of mice with a conditional Foxp2 null allele. Genesis 45, 440-446

38 Groszer, M. et al. (2008) Impaired synaptic plasticity and motor learning in mice with a point mutation implicated in human speech deficits. Curr. Biol. 18, 354-362

39 Fujita, E. et al. (2008) Ultrasonic vocalization impairment of Foxp2 $(\mathrm{R} 552 \mathrm{H})$ knockin mice related to speech-language disorder and abnormality of Purkinje cells. Proc. Natl. Acad. Sci. U. S. A. 105, 3117-3122

40 Vernes, S.C. et al. (2006) Functional genetic analysis of mutations implicated in a human speech and language disorder. Hum. Mol. Genet. $15,3154-3167$

$41 \mathrm{Shu}$, W. et al. (2007) Foxp2 and Foxp1 cooperatively regulate lung and esophagus development. Development 134, 1991-2000

42 Corrales, J.D. et al. (2006) The level of sonic hedgehog signaling regulates the complexity of cerebellar foliation. Development 133, 1811-1821

43 Feuk, L. et al. (2006) Absence of a paternally inherited FOXP2 gene in developmental verbal dyspraxia. Am. J. Hum. Genet. 79, 965-972

44 Zeesman, S. et al. (2006) Speech and language impairment and oromotor dyspraxia due to deletion of $7 \mathrm{q} 31$ that involves FOXP2 Am. J. Med. Genet. A. 140, 509-514 
45 Shriberg, L.D. et al. (2006) Speech, prosody, and voice characteristics of a mother and daughter with a $7 ; 13$ translocation affecting FOXP2. $J$. Speech Lang. Hear. Res. 49, 500-525

46 Lennon, P.A. et al. (2007) Deletion of 7q31.1 supports involvement of FOXP2 in language impairment: clinical report and review. Am. J. Med. Genet. A. 143A, 791-798

47 Ehret, G. (2005) Infant rodent ultrasounds-a gate to the understanding of sound communication. Behav. Genet. 35, 19-29

48 Holy, T.E. and Guo, Z. (2005) Ultrasonic songs of male mice. PLoS Biol. 3, e386

49 Branchi, I. et al. (2001) Ultrasonic vocalisation emitted by infant rodents: a tool for assessment of neurobehavioural development. Behav. Brain Res. 125, 49-56

50 Vargha-Khadem, F. et al. (1998) Neural basis of an inherited speech and language disorder. Proc. Natl. Acad. Sci. U. S. A. 95, 12695-12700

51 Middleton, F.A. and Strick, P.L. (2000) Basal ganglia and cerebellar loops: motor and cognitive circuits. Brain Res. Brain Res. Rev. 31, 236250

52 De Bono, J.P. et al. (2006) Novel quantitative phenotypes of exercise training in mouse models. Am. J. Physiol. Regul. Integr. Comp. Physiol. 290, R926-R934

53 Zucker, R.S. and Regehr, W.G. (2002) Short-term synaptic plasticity. Annu. Rev. Physiol. 64, 355-405

54 Gerdeman, G.L. et al. (2002) Postsynaptic endocannabinoid release is critical to long-term depression in the striatum. Nat. Neurosci. 5, 446451

55 Yin, H.H. et al. (2006) The role of protein synthesis in striatal long-term depression. J. Neurosci. 26, 11811-11820

56 Enard, W. et al. (2002) Molecular evolution of FOXP2, a gene involved in speech and language. Nature 418, 869-872

57 Zhang, J. et al. (2002) Accelerated protein evolution and origins of human-specific features: Foxp2 as an example. Genetics 162, 18251835

58 Zeigler, P. and Marler, P., eds (2008) The Neuroscience of Birdsong, Cambridge University Press

59 Scharff, C. and Nottebohm, F. (1991) A comparative study of the behavioral deficits following lesions of various parts of the zebra finch song system: implications for vocal learning. J. Neurosci. 11, $2896-2913$

60 Jarvis, E.D. et al. (1998) For whom the bird sings: context-dependent gene expression. Neuron 21, 775-788

61 Teramitsu, I. and White, S.A. (2006) FoxP2 regulation during undirected singing in adult songbirds. J. Neurosci. 26, 7390-7394

62 Miller, J.E. et al. (2008) Birdsong decreases protein levels of FoxP2, a molecule required for human speech. J. Neurophysiol. 100, 2015-2025

63 Lombardino, A.J. et al. (2006) Expression profiling of intermingled long-range projection neurons harvested by laser capture microdissection. J. Neurosci. Methods 157, 195-207

64 Haesler, S. et al. (2007) Incomplete and inaccurate vocal imitation after knockdown of FoxP2 in songbird basal ganglia nucleus Area X. PLoS Biol. 5, e321

65 Farries, M.A. et al. (2005) Evidence for "direct" and "indirect" pathways through the song system basal ganglia. J. Comp. Neurol. 484, 93-104

66 Kozhevnikov, A.A. and Fee, M.S. (2007) Singing-related activity of identified HVC neurons in the zebra finch. J. Neurophysiol. 97, 42714283
67 Schultz, W. (2007) Behavioral dopamine signals. Trends Neurosci. 30, 203-210

68 Enard, W. et al. A humanized version of Foxp2 affects cortico-basal ganglia circuits in mice. Cell (in press)

69 Bonkowsky, J.L. et al. (2008) Domain-specific regulation of foxP2 CNS expression by lef1. BMC Dev. Biol. 8, 103

70 Schroeder, D.I. and Myers, R.M. (2008) Multiple transcription start sites for FOXP2 with varying cellular specificities. Gene 413, 42-48

$71 \mathrm{Li}, \mathrm{S}$. et al. (2004) Transcriptional and DNA binding activity of the Foxp1/2/4 family is modulated by heterotypic and homotypic protein interactions. Mol. Cell. Biol. 24, 809-822

72 Vernes, S.C. et al. Assessing the impact of FOXP1 mutations on developmental verbal dyspraxia. Eur. J. Hum. Genet. (in press)

73 Zhou, B. et al. (2008) Foxp2 inhibits Nkx2.1-mediated transcription of SP-C via interactions with the Nkx2. 1 homeodomain. Am. J. Respir. Cell Mol. Biol. 38, 750-758

74 Fisher, S.E. et al. (1998) Localisation of a gene implicated in a severe speech and language disorder. Nat. Genet. 18, 168-170

75 Lai, C.S. et al. (2000) The SPCH1 region on human 7q31: genomic characterization of the critical interval and localization of translocations associated with speech and language disorder. Am. J. Hum. Genet. 67, 357-368

76 Newbury, D.F. et al. (2005) Genetic influences on language impairment and phonological short-term memory. Trends Cogn. Sci. 9, 528-534

77 Tomblin, J.B. et al. (1997) Prevalence of specific language impairment in kindergarten children. J. Speech Lang. Hear. Res. 40, 1245-1260

78 Bishop, D.V. et al. (1995) Genetic basis of specific language impairment: evidence from a twin study. Dev. Med. Child Neurol. $37,56-71$

79 Bartlett, C.W. et al. (2002) A major susceptibility locus for specific language impairment is located on 13q21. Am. J. Hum. Genet. 71, 45-55

80 Newbury, D.F. et al. (2002) FOXP2 is not a major susceptibility gene for autism or specific language impairment. Am. J. Hum. Genet. 70, 13181327

81 Coop, G. et al. (2008) The timing of selection at the human FOXP2 gene. Mol. Biol. Evol. 25, 1257-1259

82 Krause, J. et al. (2007) The derived FOXP2 variant of modern humans was shared with Neandertals. Curr. Biol. 17, 1908-1912

83 Weaver, T.D. et al. (2008) Close correspondence between quantitativeand molecular-genetic divergence times for Neandertals and modern humans. Proc. Natl. Acad. Sci. U. S. A. 105, 4645-4649

84 Fisher, S.E. (2006) Tangled webs: tracing the connections between genes and cognition. Cognition 101, 270-297

85 Webb, D.M. and Zhang, J. (2005) FoxP2 in song-learning birds and vocal-learning mammals. J. Hered. 96, 212-216

$86 \mathrm{Li}$, G. et al. (2007) Accelerated FoxP2 evolution in echolocating bats. PLoS One 2, e900

87 Theunissen, F.E. and Shaevitz, S.S. (2006) Auditory processing of vocal sounds in birds. Curr. Opin. Neurobiol. 16, 400-407

88 Williams, H. and Nottebohm, F. (1985) Auditory responses in avian vocal motor neurons: a motor theory for song perception in birds. Science 229, 279-282

89 Prather, J.F. et al. (2008) Precise auditory-vocal mirroring in neurons for learned vocal communication. Nature 451, 305-310

90 Hahnloser, R.H. et al. (2002) An ultra-sparse code underlies the generation of neural sequences in a songbird. Nature 419, 65-70 